\title{
The population genetics of the self-incompatibility polymorphism in Papaver rhoeas. IX. Evidence of an extra effect of selection acting on the $S$-locus
}

\author{
M. J. LAWRENCE* \& V. E. FRANKLIN-TONG \\ Wolfson Laboratory for Plant Molecular Biology, School of Biological Sciences, University of Birmingham, \\ Birmingham B15 2TT, U.K.
}

\begin{abstract}
Investigation of the segregation ratios of the self-incompatibility $(S-)$ alleles in three sets of full-sib families of Papaver rhoeas shows that these ratios depart significantly from Mendelian expectation. This indicates that the $S$-locus is subject to an extra effect of selection over and above that due to incompatibility in these families, and suggests that this is probably the chief cause of the unequal $S$ allele frequencies observed in the R102 and the other natural populations we have examined. Since, however, the selective advantage of an allele depends on the allele with which it is segregating in all of the families examined, the relationship between these results and the unequal $S$-allele frequencies in populations appears to be complex. Furthermore, while in one family the extra effect of selection appears to be of the zygotic type, in the others it is of the gametic type - which, in most, involves the female gametophyte rather than the pollen. It is argued that the extra effect of selection is due to the linkage of the $S$-gene to one or more genes that are the chief target of this selection, rather than to pleiotropy. Though it is suggested that among candidates for linkage are genes controlling seed dormancy and albinism, the detection of an extra effect of selection acting on the female gametophyte and, in one case, on the pollen, implies that other genes of as yet unknown effect are more likely to be involved.
\end{abstract}

Keywords: linkage disequilibrium, Papaver rhoeas, polymorphism, population genetics, segregation ratios, self-incompatibility.

\section{Introduction}

In the previous paper, we discussed the hypothesis that the unequal $S$-allele frequencies in the British populations of Papaver rhoeas we have examined were caused by a sampling effect over and above that due to drift. Despite the fact that it can take many generations before a population achieves equilibrium with respect to the frequencies of the alleles it contains after these have been perturbed, it was concluded that the size of these British populations was such as to limit opportunities for the kind of occasional sampling effect envisaged in this hypothesis. Thus, with the possible exception that non-random distribution of progeny size might inflate the variance of allele frequency caused by drift to an extent that could account for the magnitude of the unequal allele frequencies observed, the hypothesis that these are caused by some sort of samp-

\footnotetext{
${ }^{*}$ Correspondence.
}

ling effect of a kind to be expected in a species occurring in distributed habitats now appears somewhat less likely than before (Lawrence et al., 1994).

In this paper, we consider the alternative hypothesis that these unequal $S$-allele frequencies are caused by an extra effect of selection acting on some of the alleles in these populations, over and above that due to incompatibility. When this hypothesis was originally put forward, it was thought that this extra effect of selection could be due either to a pleiotropic property of these alleles or to their linkage to another gene that was the chief target of this extra effect of selection (Campbell \& Lawrence, 1981). However, since it has now been established that different $S$-alleles occur at a relatively high frequency in different populations, the possibility that this extra effect of selection is due to pleiotropy appears to have been effectively ruled out (Lawrence et al., 1993). Hence, if the unequal $S$-allele frequencies in our British populations are, in fact, caused by an extra effect of selection acting on the 
locus, this is more likely to be due to its linkage to another gene that is the chief target of this selection.

As was pointed out in our previous paper (Lawrence et al., 1994), it should be possible, in principle, to test this hypothesis by examining segregation ratios in families that are segregating for one allele, $S_{\mathrm{I}}$ say, that occurs at a relatively high frequency in the population from which the parents of these families were derived, and three alleles, $S_{j}, S_{\mathrm{k}}$ and $S_{1}$, which occur at a relatively low frequency in that population. Suppose the cross giving rise to the family is $S_{\mathrm{I}} S_{\mathrm{j}} \times S_{\mathrm{k}} S_{1}$. Then, if the relatively high frequency of $S_{\mathrm{I}}$ in that population is due to the fact that it is subject to a favourable additional effect of selection compared with $S_{1}, S_{\mathrm{k}}$ and $S_{1}$, plants of genotype $S_{\mathrm{I}} S_{\mathrm{k}}$ and $S_{\mathrm{I}} S_{1}$ should occur in greater numbers in the progeny than those of genotype $S_{\mathrm{j}} S_{\mathrm{k}}$ or $S_{\mathrm{j}} S_{1}$. Furthermore, if the cross were made in reciprocal, it should be possible to find out whether this selection was of the gametic or zygotic type, the reciprocals being expected to be heterogeneous with respect to disturbed ratios with the former, but homogeneous with the latter.

In practice, it could be difficult to detect selection in families of a realistic size unless this selection was quite strong. In the course of carrying out the cross-classification of the $S$-alleles of each sample taken from our British populations against those of the others, we have raised most of the families produced by crossing the plants classified in the original diallel experiments (Campbell \& Lawrence, 1981; Lawrence \& O'Donnell, 1981) and have not infrequently found that the ratios in the four-class families deviated significantly from the expected 1:1:1:1 (Lawrence et al., 1993). Since, however, no more than 20 plants were ever raised in these families, a number which gives a high probability of a family containing at least one plant of each class, assuming that these classes are equally frequent (Lawrence et al., 1978), and not every plant was always fully classified in every family, these data are not suit- able for our present purpose. Nevertheless, the results obtained from this large number of families did at least suggest that it might be possible to detect an extra effect of selection acting on the $S$-locus if a larger number of plants were to be raised in appropriate families.

In this paper, we report the results obtained from three experiments which suggest that the locus is, indeed, subject to an extra effect of selection, over and above that caused by incompatibility.

\section{Materials and methods}

\section{The first experiment}

The parents of the two families used in the first experiment were four of the 36 plants whose incompatibility genotype had been determined in the R102 diallel experiment carried out by Lawrence and O'Donnell (1981). Thus, the parents of the first, Family 9, were plants 4 and 9 , whose genotypes were $S_{1} S_{5}$ and $S_{10} S_{11}$, respectively, and those of the second, Family 11 , were plants 2 and 28, whose genotypes were $S_{3} S_{4}$ and $S_{16} S_{19}$, respectively (Table 1 ). $S_{1}$ and $S_{16}$ were two of the three most frequently occurring alleles in the R102 sample, each occurring seven times, whereas $S_{3}, S_{4}, S_{5}$ and $S_{10}$ occurred only three times, $S_{19}$ twice and $S_{11}$ once (Table 4 of Lawrence \& O'Donnell, 1981, with the corrections shown in Table 2 of Lawrence et al., 1993). Each of these families, therefore, was of the type described in the previous section in that, of the four alleles expected to segregate in them, one occurred at a relatively high frequency in the R102 sample and the others at a relatively low frequency.

The intention was to raise 50 plants of each reciprocal in each family. In order to do this, it was first necessary to break the innate dormancy of the seed, which is a characteristic of all seed of this species of wild-type origin. Our standard procedure is as follows.

Table 1 The pair of R102 families used in the first experiment; the pedigree numbers cross-reference the parents of these families to Table 3 of Lawrence and O’Donnell (1981)

\begin{tabular}{|c|c|c|c|c|c|c|}
\hline & Pedigree & Parental & \multicolumn{4}{|c|}{ Genotypes in progeny } \\
\hline $9 \mathrm{~A}$ & $4 \times 9$ & $S_{1} S_{5} \times S_{10} S_{11}$ & \multirow[b]{2}{*}{$S_{1} S_{10}$} & \multirow[b]{2}{*}{$S_{1} S_{11}$} & \multirow[b]{2}{*}{$S_{5} S_{10}$} & \multirow[b]{2}{*}{$S_{5} S_{11}$} \\
\hline $9 \mathrm{~B}$ & $9 \times 4$ & $S_{10} S_{11} \times S_{1} S_{5}$ & & & & \\
\hline $11 \mathrm{~A}$ & $2 \times 28$ & $S_{3} S_{4} \times S_{16} S_{19}$ & \multirow[b]{2}{*}{$S_{3} S_{16}$} & \multirow[b]{2}{*}{$S_{3} S_{19}$} & \multirow[b]{2}{*}{$S_{4} S_{16}$} & \multirow[b]{2}{*}{$S_{4} S_{19}$} \\
\hline $11 \mathrm{~B}$ & $28 \times 2$ & $S_{16} S_{19} \times S_{3} S_{4}$ & & & & \\
\hline
\end{tabular}


Dry seed is immersed in concentrated sulphuric acid for 25-90 s and then quickly transferred to a sintered glass funnel where it is thoroughly washed in tap water. The seed is then transferred to a $6 \mathrm{~cm}$ Petri dish, where it is allowed to imbibe water from filter paper for $24 \mathrm{~h}$ at room temperature, before being transferred to $3-5^{\circ} \mathrm{C}$ for six days. The treatment is completed by returning the dishes to room temperature for a further $24 \mathrm{~h}$, after which the seed that has germinated is sown with a fine paintbrush on the surface of John Innes Potting Compost No. 1 in 'Jiffy' pots, three or four seeds being sown in each pot. The duration of the acid treatment required to give a high germination percentage varies with seed lots and seasons, so that it is necessary to conduct a small-scale preliminary trial in order to determine the optimum duration of this treatment for the seed used in any one experiment. With care, it is usually possible to raise the germination percentage of the seed from 2-5 per cent, the percentage obtained without treatment, to $80-100$ per cent for most seed lots.

After ten days, seedlings are thinned, at random, to leave one in each pot, after which they are held in the glasshouse for approximately two weeks before being transferred to an unheated frame in order to harden them off prior to their being transplanted into open ground.

The preliminary classification of the plants of these families was carried out using the procedure described by Lawrence et al. (1993), and the assignation of incompatibility genotypes to classes was accomplished by crossing plants from each of the four classes in each family to plants from other families that contained the same alleles as those of Families 9 or 11 that were included in this experiment for this purpose.

\section{The second experiment}

The purpose of the second experiment was to examine segregation ratios in families produced from all three of the fully compatible crosses that are possible in a pedigree segregating for four alleles. The first of these, Family 9.0, was the same as Family 9 of the previous experiment, seed being taken from the same packet as that used to raise the plants of Family 9. The seed used to raise the other two families was obtained by crossing fully compatible plants from Family 9 of the first experiment, these crosses producing the seed of Families 9.3 and 9.4 (Table 2). Fifty plants were raised from the seed from each reciprocal of each of the three crosses. For reasons that will emerge, an attempt was made to break the dormancy of this seed only partially by using a sub-optimal acid treatment. All other technical details were the same as for the first experiment.

\section{The third experiment}

The ten families of the third experiment belonged to a pedigree established by crossing a pair of plants that were raised from the seed of Sutton's 'Shirley' cultivar of $P$. rhoeas. Though the seed of this cultivar lacks the dormancy of that from natural populations, its individuals are as self-incompatible as those raised from seed taken from natural populations. Three $S$-alleles were segregating in these families, which were arbitrarily labelled as $S_{1}, S_{2}$ and $S_{3}$. Though it was subsequently discovered that $S_{3}$ is identical to the R102 allele $S_{11}$ of Family 9 of the previous experiments, the counterparts of $S_{1}$ and $S_{2}$ in this population are not yet known. It was possible to raise and classify a much larger number of plants than in the previous experi-

Table 2 The genotypes of the progeny occurring $(\sqrt{ })$ in each of the three families raised in the second experiment

\begin{tabular}{|c|c|c|c|c|c|c|c|}
\hline \multirow[b]{2}{*}{ Family } & \multirow{2}{*}{$\begin{array}{l}\text { Parental } \\
\text { genotype }\end{array}$} & \multicolumn{6}{|c|}{ Genotypes among progeny } \\
\hline & & $S_{1} S_{5}$ & $S_{1} S_{10}$ & $S_{1} S_{11}$ & $S_{5} S_{10}$ & $S_{5} S_{11}$ & $S_{10} S_{11}$ \\
\hline 9.0 & $\begin{array}{l}S_{1} S_{5} \times S_{10} S_{11} \\
S_{10} S_{11} \times S_{1} S_{5}\end{array}$ & . & J & J & J & J & ${ }^{\circ}$ \\
\hline 9.3 & $\begin{array}{l}S_{1} S_{10} \times S_{5} S_{11} \\
S_{5} S_{11} \times S_{1} S_{10}\end{array}$ & 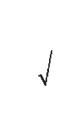 & . & $\sqrt{ }$ & J & . & J \\
\hline 9.4 & $\begin{array}{l}S_{5} S_{10} \times S_{1} S_{11} \\
S_{1} S_{11} \times S_{5} S_{10}\end{array}$ & J & $\sqrt{ }$ & . & $\cdot$ & $\sqrt{ }$ & J \\
\hline
\end{tabular}


ments, partly because only two incompatibility classes occurred in these families, but also because the use of pollen from plants of known incompatibility genotype, stored from previous seasons, increased the efficiency and speed of classification(Tong, 1986).

The chief purpose of raising these families over a number of seasons was to provide pollen and stigmas for our investigation of the molecular biology of selfincompatibility in this species (Franklin-Tong \& Franklin, 1992; Franklin-Tong et al., 1988, 1989, 1990, 1991, 1993; Foote et al., 1994). These families, unlike those of the previous experiments, have effectively been chosen at random so far as the investigation of an extra effect of selection acting on the $S$-locus is concerned. The results presented here are a subset of a large body of data accumulated over eight seasons of work, with the requirement that no family should contain fewer than 30 fully classified plants.

\section{Results}

\section{The first experiment}

The results obtained from Family 9 are shown in Table 3 and those from Family 11 in Table 4. Turning first to those from Family 9, it is immediately apparent that the

Table 3 (a) The results obtained from Family 9 and (b) the analysis of these results; the cross giving rise to $9 \mathrm{~A}$ was $S_{1} S_{5} \times S_{10} S_{11}$ and for the reciprocal 9B, $S_{10} S_{11} \times S_{1} S_{5}$

(a)

\begin{tabular}{lccccc}
\hline \multicolumn{5}{c}{ Progeny } \\
\cline { 2 - 4 } Family & $S_{1} S_{10}$ & $S_{1} S_{11}$ & $S_{5} S_{10}$ & $S_{5} S_{11}$ & Totals \\
\hline $9 \mathrm{~A}$ & 16 & 15 & 5 & 5 & 41 \\
$9 \mathrm{~B}$ & 18 & 13 & 8 & 9 & 48 \\
$9 \mathrm{~A}+9 \mathrm{~B}$ & 34 & 28 & 13 & 14 & 89 \\
\hline
\end{tabular}

(b)

\begin{tabular}{lcc}
\hline Item & d.f. & $\chi^{2}$ \\
\hline Deviation & 3 & $14.596^{* * *}$ \\
$S_{1}$ versus $S_{5}$ & 1 & $13.764^{* * * *}$ \\
Remainder & 2 & 0.832 \\
Heterogeneity $\dagger$ & 3 & 1.555 \\
$S_{1}$ versus $S_{5} \dagger$ & 1 & 1.272 \\
Remainder & 2 & 0.301 \\
\hline
\end{tabular}

${ }^{* *} P<0.01,{ }^{* * *} P<0.001$.

$\Varangle$ Contingency $\chi^{2}$ values, which are the appropriate tests when the null hypothesis of equal numbers in each class has been rejected on the totals. numbers of plants in the four classes depart significantly from the expected $1: 1: 1: 1$ ratio. This departure is due solely to the excess of plants containing the $S_{1}$ allele over those containing the alternative allele, $S_{5}$, the ratio $S_{1}: S_{5}$ being $62: 27$. Furthermore, since the reciprocals are homogeneous in this respect, the extra effect of selection acting on the $S$-locus in this family appears to be of the zygotic kind. It will be recalled that $S_{1}$ was one of the three most frequently occurring alleles in the R102 population from which the parents of this family originated. The results obtained from this family, therefore, are consistent with the hypothesis that the unequal allele frequencies in this population are due to an extra effect of selection acting on the locus over and above that due to incompatibility.

The results obtained from Family 11 (Table 4) are similar to those from Family 9 in that there is a highly significant departure of the numbers of plants in the four classes from the expected 1:1:1:1 ratio and that the reciprocals are homogeneous in this respect - suggesting, again, that the extra effect of selection is of the zygotic kind. In this case, however, this departure is due mainly to an excess of plants containing the $S_{3}$ allele compared with those containing the alternative

Table 4 (a) The results obtained from Family 11 and (b) the analysis of these results; the cross giving rise to $11 \mathrm{~A}$ was $S_{3} S_{4} \times S_{16} S_{19}$ and for the reciprocal 11B, $S_{16} S_{19} \times S_{3} S_{4}$

(a)

\begin{tabular}{lrrrrr}
\hline & \multicolumn{4}{c}{ Progeny } & \\
\cline { 2 - 4 } Family & $S_{3} S_{16}$ & $S_{3} S_{19}$ & $S_{4} S_{16}$ & $S_{4} S_{19}$ & Totals \\
\hline $11 \mathrm{~A}$ & 8 & 24 & 2 & 3 & 37 \\
$11 \mathrm{~B}$ & 18 & 19 & 6 & 7 & 50 \\
$11 \mathrm{~A}+11 \mathrm{~B}$ & 26 & 43 & 8 & 10 & 87 \\
\hline
\end{tabular}

(b)

\begin{tabular}{lcc}
\hline Item & d.f. & $\chi^{2}$ \\
\hline Deviation & 3 & $36.632^{* * *}$ \\
$S_{3}$ versus $S_{4}$ & 1 & $29.897^{* * *}$ \\
$S_{16}$ versus $S_{19}$ & 1 & $4.149^{*}$ \\
Remainder & 1 & 2.586 \\
Heterogeneity $\dagger$ & 3 & 6.224 \\
$S_{3}$ versus $S_{4} \dagger$ & 1 & 2.020 \\
$S_{16}$ versus $S_{19} \dagger$ & 1 & $3.929^{*}$ \\
Remainder & 1 & 3.495 \\
\hline
\end{tabular}

${ }^{*} P<0.05, * * * P<0.001$.

$†$ Contingency $\chi^{2}$ values, which are the appropriate tests when the null hypothesis of equal numbers in each class has been rejected on the totals. 
$S_{4}$ allele, the ratio $S_{3}: S_{4}$ being $69: 18$. In addition, there is also a just significant excess of plants containing $S_{19}$ over those containing $S_{16}$, the ratio of the $S_{19}: S_{16}$ being $53: 34$, though this disturbance is not quite homogeneous over reciprocals.

It is difficult to know what to make of these results. The alleles in excess, $S_{3}$ and $S_{19}$, are not among the most frequent in the population from which the parents were obtained, but the allele which is the most frequent of the four in the R102 population, $S_{16}$, is actually the least frequent in the family. Furthermore, since $S_{3}$ is not the alternative allele to $S_{16}$, it is impossible to explain the high frequency of the $S_{3}$ in the family in terms of a recombinational event between $S_{16}$ and a gene which is the chief target of the extra effect of selection (though this possibility could go some way to explaining why plants containing $S_{19}$ occur more frequently in the family than those containing $\left.S_{16}\right)$. Thus, while the results from this family indicate that the locus is subject to an extra effect of selection, it is not possible, as with those from Family 9, to relate them directly to the results obtained from our survey of the number and frequency of the alleles in the R102 population (Lawrence \& O’Donnell, 1981).

Because this experiment was designed to investigate the possibility that the $S$-locus was subject to an extra effect of selection, special care was taken to avoid introducing any extraneous selection during the course of the experiment. Thus, all plants that flowered were fully classified and the only loss of material that occurred between the time when the seed was sown and when the plants were classified was due to the unfortunate destruction of 24 plants by pigeons shortly after they had been transplanted into open ground. Assuming, not unreasonably, that this predation was not selective with respect to the $S$-locus, the zygotic selection detected in both of these families must have occurred sometime between the fertilization of the ovules from which the seed used to raise these plants developed and this seed being sown.

It is possible that the extra effect of selection is due to differential mortality between zygotes while they are in the ovary. At the time, however, it was thought that a more likely cause of the disturbed segregation ratios observed in these families was that the $S$-gene was linked to a gene that controls seed dormancy since, in initiating this experiment, some difficulty was experienced in germinating the seed, presumably because a sub-optimal duration of acid treatment was used to break the innate dormancy of the seed, with the result that the percentage germination obtained was less than usual. The advantage of this hypothesis is that it is, in principle, testable. Thus, if the disturbed segregation ratios observed in these families are due to differential germination of the seed, it should be possible to eliminate this disturbance if the seed is treated in a way that results in 100 per cent germination. Conversely, if this is not the case we would expect the magnitude of this disturbance to be negatively related to germination percentage of the seed such that it increases as the germination percentage declines. This is a possibility which was investigated in the second experiment.

\section{The second experiment}

For reasons that will become apparent, it is convenient to consider first the results obtained from Family 9.0 (Table 5). While the total numbers of plants falling into each of the four classes appear to be entirely consistent with Mendelian expectation, the ratio $S_{1}: S_{5}$ is just significantly heterogeneous over reciprocals, this ratio being $27: 15$ in $9.0 \mathrm{~A}$, but $19: 27$ in $9.0 \mathrm{~B}$, though neither of these ratios departs significantly from the expected 1:1 ratio. The disturbance, therefore, that was detected in this family in a previous season, appears to be absent in the present data.

Analysis of the results from this family pooled over seasons, however, reveals that there is a highly significant overall excess of plants containing $S_{1}$ compared with those containing $S_{5}$, the ratio $S_{1}: S_{5}$ being $108: 69$, and that this comparison is only marginally heterogenous over seasons (Table 6a). This evidence

Table 5 (a) The results obtained from Family 9.0 and (b) the analysis of these results

(a)

\begin{tabular}{lrrrrr}
\hline & \multicolumn{5}{c}{ Progeny } \\
\cline { 2 - 5 } Family & $S_{1} S_{10}$ & $S_{1} S_{11}$ & $S_{5} S_{10}$ & $S_{5} S_{11}$ & Totals \\
\hline $9.0 \mathrm{~A}$ & 14 & 13 & 10 & 5 & 42 \\
$9.0 \mathrm{~B}$ & 9 & 10 & 9 & 18 & 46 \\
$9.0 \mathrm{~A}+9.0 \mathrm{~B}$ & 23 & 23 & 19 & 23 & 88 \\
\hline
\end{tabular}

(b)

\begin{tabular}{lcc}
\hline Item & d.f. & $\chi^{2}$ \\
\hline Deviation & 3 & 0.545 \\
$S_{1}$ versus $S_{5}$ & 1 & 0.182 \\
Remainder & 2 & 0.363 \\
Heterogeneity & 3 & $9.079^{*}$ \\
$S_{1}$ versus $S_{5}$ & 1 & $4.638^{*}$ \\
Remainder & 2 & 4.441 \\
\hline
\end{tabular}

$* P<0.05$ 
suggests that the extra effect of selection acting on the $S$-locus is of the zygotic kind. However, analysis of the results from each reciprocal pooled over seasons shows that the disturbance is confined to reciprocal A, where the ratio $S_{1}: S_{5}$ is $58: 25$ (Table $6 \mathrm{~b}$ ). Furthermore, not only is there no significant overall departure of the ratio $S_{1}: S_{5}$ from the expected 1:1 ratio in reciprocal $\mathrm{B}$, where the observed ratio is only $50: 44$, but this ratio is marginally heterogeneous over seasons (Table 6c). On this evidence, therefore, the extra effect of selection appears to be of the gametic rather than the zygotic kind. Unfortunately, it is not possible to distinguish unambiguously between these

Table 6 The analysis of the results obtained from Family 9 (a) pooled over seasons and reciprocals $(9+9.0)$; (b) pooled over reciprocal $\mathrm{A}(9 \mathrm{~A}+9.0 \mathrm{~A})$; and $(\mathrm{c})$ pooled over reciprocal $\mathrm{B}(9 \mathrm{~B}+9.0 \mathrm{~B})$

(a)

\begin{tabular}{lcl}
\hline Item & d.f. & \multicolumn{1}{c}{$\chi^{2}$} \\
\hline Deviation & 3 & $9.282^{*}$ \\
$S_{1}$ versus $S_{5}$ & 1 & $8.593^{* *}$ \\
Remainder & 2 & 0.690 \\
Heterogeneity $\dagger$ & 3 & $7.931^{*}$ \\
$S_{1}$ versus $S_{5} \dagger$ & 1 & $5.161^{*}$ \\
Remainder & 2 & 2.476 \\
\hline
\end{tabular}

(b)

\begin{tabular}{lcc}
\hline Item & d.f. & $\chi^{2}$ \\
\hline Deviation & 3 & $13.819^{* * *}$ \\
$S_{1}$ versus $S_{5}$ & 1 & \\
Remainder & 2 & $13.120^{* * * *}$ \\
Heterogeneity $\dagger$ & 3 & 0.698 \\
$S_{1}$ versus $S_{5} \dagger$ & 1 & 1.931 \\
Remainder & 2 & 1.264 \\
\hline
\end{tabular}

(c)

\begin{tabular}{lcc}
\hline Item & d.f. & $\chi^{2}$ \\
\hline Deviation & 3 & 2.851 \\
$S_{1}$ versus $S_{5}$ & 1 & 0.383 \\
Remainder & 2 & 2.468 \\
Heterogeneity & 3 & 7.273 \\
$S_{1}$ versus $S_{5}$ & 1 & $5.091^{*}$ \\
Remainder & 2 & 2.182 \\
\hline
\end{tabular}

${ }^{*} P<0.05,{ }^{* *} P<0.01,{ }^{* * * * *} P<0.001$.

†Contingency $\chi^{2}$ values, which are the appropriate tests when the null hypothesis of equal numbers in each class has been rejected on the totals. alternative interpretations of these data on the evidence from this family alone. In view of the results obtained from the remaining pair of families in this experiment, however, we prefer the second of these alternatives; namely, that the disturbance in this family is due to an extra effect of selection acting on the $S$ locus of the gametic kind.

The results obtained from Families 9.3 and 9.4 are shown in Tables 7 and 8 , respectively. In the case of Family 9.3, the sole cause of departure from Mendelian expectation is an excess of plants containing $S_{11}$ in reciprocal $\mathrm{B}$ compared with those containing the alternative allele, $S_{5}$, the ratio $S_{5}: S_{11}$ being 11:27. In Family 9.4, on the other hand, disturbed segregation ratios can be detected only in reciprocal $\mathrm{A}$, where the ratio $S_{1}: S_{11}$ is $40: 9$. In each of these families, the reciprocals are significantly heterogeneous with respect to these ratios, so that in both, the disturbance appears to be of the gametic kind.

There are three further points worth making about the results of this second experiment. First, though it was intended to classify 50 plants of each reciprocal in each of the three families, some losses occurred. Some

Table 7 (a) The results obtained from Family 9.3, (b) the analysis of the results from reciprocal $A$ and $(\mathrm{c})$ the analysis of those from reciprocal $\mathrm{B}$; the cross giving rise to reciprocal A was $S_{1} S_{10} \times S_{5} S_{11}$ and that for B was $S_{5} S_{11} \times S_{1} S_{10}$ (a)

\begin{tabular}{lrrrrr}
\hline \multicolumn{5}{c}{ Progeny } & \\
\cline { 2 - 5 } Family & $S_{1} S_{5}$ & $S_{1} S_{11}$ & $S_{5} S_{10}$ & $S_{10} S_{11}$ & Totals \\
\hline $9.3 \mathrm{~A}$ & 11 & 5 & 7 & 5 & 28 \\
$9.3 \mathrm{~B}$ & 7 & 13 & 4 & 14 & 38 \\
$9.3 \mathrm{~A}+9.3 \mathrm{~B}$ & 18 & 18 & 11 & 19 & 66 \\
\hline
\end{tabular}

(b).

\begin{tabular}{lcc}
\hline Item & d.f. & $\chi^{2}$ \\
\hline Deviation & 3 & 3.429 \\
$S_{5}$ versus $S_{11}$ & 1 & 2.286 \\
Remainder & 2 & 1.143 \\
\hline
\end{tabular}

(c)

\begin{tabular}{lcc}
\hline Item & d.f. & \multicolumn{1}{c}{$\chi^{2}$} \\
\hline Deviation & 3 & 7.263 \\
$S_{5}$ versus $S_{11}$ & 1 & $6.737^{* *}$ \\
Remainder & 2 & 0.526 \\
\hline
\end{tabular}

$* * P<0.01$. 
Table 8 (a) The results obtained from Family 9.4, (b) the analysis of the results from reciprocal $\mathrm{A}$ and (c) the analysis of those from reciprocal $B$; the cross giving rise to reciprocal A was $S_{5} S_{10} \times S_{1} S_{11}$ and that for $\mathrm{B}$ was $S_{1} S_{11} \times S_{5} S_{10}$

(a)

\begin{tabular}{lrrrrr}
\hline & \multicolumn{5}{c}{ Progeny } \\
\cline { 2 - 5 } Family & $S_{1} S_{5}$ & $S_{1} S_{10}$ & $S_{5} S_{11}$ & $S_{10} S_{11}$ & Totals \\
\hline $9.4 \mathrm{~A}$ & 14 & 26 & 5 & 4 & 49 \\
$9.4 \mathrm{~B}$ & 9 & 11 & 16 & 12 & 48 \\
$9.4 \mathrm{~A}+9.4 \mathrm{~B}$ & 23 & 37 & 21 & 16 & 97 \\
\hline
\end{tabular}

(b)

\begin{tabular}{lcc}
\hline Item & d.f. & $\chi^{2}$ \\
\hline Deviation & 3 & $25.531^{* * *}$ \\
$S_{1}$ versus $S_{11}$ & 1 & $19.612^{* * *}$ \\
Remainder & 2 & 5.919 \\
\hline
\end{tabular}

(c)

\begin{tabular}{lcc}
\hline Item & d.f. & $\chi^{2}$ \\
\hline Deviation & 3 & 2.167 \\
$S_{1}$ versus $S_{11}$ & 1 & 1.333 \\
Remainder & 2 & 0.834 \\
\hline
\end{tabular}

*** $P<0.001$ of these losses were due to the fact that in each family a proportion of the seed gave rise to albino seedlings (Table 9), though the majority of these were replaced by wild-type siblings. The most serious losses occurred in Family 9.3 which contained a number of plants with aberrant phenotypes (Table 9), due, no doubt, to the fact that this family (and Family 9.4) were the product of one round of inbreeding by sib-mating. All but two of the plants with dissected petals or that were male sterile were completely classified, the male sterile plants being used as females in the diagnostic pollinations. However, 27 of the plants in this family bore leaves which were glossy, rather than grey-green, and which were relatively undissected like those on the seedlings of young plants. Furthermore, all of these plants bore only a single flower which was frequently male sterile. Only four of these plants of 'juvenile' form could be classified for their incompatibility phenotype. The chief point here is that the losses of material which occurred in this experiment were due to natural causes rather than to those arising from our experimental procedure.

Second, it will be recalled that because of the results obtained from the first experiment, an attempt was made in this experiment to only partially break the dormancy of the seed from which the plants of the three families were raised. The germination percentages of the seed given in Table 9 show that this attempt was successful. But there appears, with one possible

Table 9 Percentage germination of seed and of albino seedlings, and, for Family 9.3 , the number of plants of aberrant phenotype

\begin{tabular}{lccccccc}
\hline Family & $n$ & \multicolumn{1}{c}{$\chi^{2}$} & $\begin{array}{c}\text { Germination } \\
(\%)\end{array}$ & $\begin{array}{c}\text { Albinos } \\
(\%)\end{array}$ & $\begin{array}{c}\text { Dissected } \\
\text { petals }\end{array}$ & $\begin{array}{c}\text { Male } \\
\text { sterile }\end{array}$ & $\begin{array}{c}\text { Juvenile } \\
\text { forms }\end{array}$ \\
\hline $9.0 \mathrm{~A}$ & 42 & 4.667 & 63.5 & 6.3 & - & - & - \\
$9.0 \mathrm{~B}$ & 46 & 4.957 & 40.0 & 13.6 & - & - & - \\
$9.3 \mathrm{~A}$ & 28 & 3.429 & 48.0 & 15.6 & $16(2)$ & $5(0)$ & $14(14)$ \\
$9.3 \mathrm{~B}$ & 38 & 7.263 & 41.5 & 12.0 & $19(0)$ & $11(0)$ & $13(9)$ \\
$9.4 \mathrm{~A}$ & 49 & 25.531 & 39.0 & 25.6 & - & - & - \\
$9.4 \mathrm{~B}$ & 48 & 2.167 & 58.5 & 6.8 & - & - & - \\
\hline
\end{tabular}

The germination and albino percentages are based on 220 treated seeds in Family $9.0 \mathrm{~B}$ and on 200 seeds in the other families.

The duration of acid treatment was $25 \mathrm{~s}$ for the seed of families $9.3 \mathrm{~A}, 9.4 \mathrm{~A}$ and $9.4 \mathrm{~B} ; 60 \mathrm{~s}$ for $9.0 \mathrm{~A}$; and $90 \mathrm{~s}$ for $9.0 \mathrm{~A}$ and $9.3 \mathrm{~B}$.

The entries in brackets for the aberrant plants of Family 9.3 show the number that could not be classified for their incompatibility phenotype.

The second column in the table shows the number of plants that were fully classified.

The third column shows the Deviation $\chi^{2}$ values testing for agreement with the Mendelian hypothesis of equal numbers of plants in the four classes. 
exception, to be no obvious negative relationship between the germination percentage of this seed and the magnitude of disturbance of the segregation ratios in these families as measured by the Deviation $\chi^{2}$. Thus, despite the fact that there is a large and significant difference between the percentage germination of the seed of Families $9.0 \mathrm{~A}$ and $9.0 \mathrm{~B}$, there was no evidence in either that the ratio of the numbers of plants in the four classes deviated from the expected Mendelian equality (Table 5). Conversely, though the germination percentages of the seed of Families 9.3A and $9.3 \mathrm{~B}$ are not significantly different, the segregation ratios are disturbed in 9.3B, but not in 9.3A (Table 7). In Family 9.4, on the other hand, the relationship between the percentage germination of the seed and the magnitude of disturbance of the segregation ratios is as expected if, as suggested in the previous section, the $S$-gene is linked to another gene which controls seed dormancy. Thus, in this family, disturbed ratios were detected only in reciprocal $\mathrm{A}$ in which the percentage germination of the seed is significantly less than that of the seed of reciprocal B (Table 8).

There is, however, a difficulty with this argument. Suppose that the disturbed ratios in these families are, in fact, caused by linkage of the $S$-gene to another gene that controls seed dormancy. Then any additional selection arising from this cause would be of the zygotic kind. However, this selection can be detected only when germination of the seed is incomplete and is expected to disturb segregation ratios increasingly as the percentage germination of the seed declines. Suppose that the percentage germination of the seed in one reciprocal of a cross is, say, 70 per cent and in the other, only 30 per cent. Then, while we would expect to observe disturbed segregation ratios in the latter, we might not in the former, unless these families were very large. But any difference between reciprocals in respect of this disturbance would be ascribed to selection of the gametic kind. Hence, differences between reciprocal families in the germination percentage of their seed could be expected, in these circumstances, to mimic gametic selection even though the true effect of this selection was, in fact, zygotic. Only if the percentage germination of the seed is similar in each reciprocal can we be reasonably confident that differences between reciprocals in their segregation ratios are likely to be due to an extra effect of selection of the gametic kind, as is arguably the case with Family 9.3. Thus, while on balance the results from this experiment suggest that an extra effect of selection of the gametic, rather than the zygotic kind, is acting on the $S$-locus, this conclusion cannot be regarded as other than tentative.

The third and final point worth making about the results of this experiment concerns the source of the disturbance detected in these families. In Family 9.0, from the data pooled over seasons, the disturbed ratios are due solely to an excess of $S_{1}$ over $S_{5}$ in reciprocal $\mathrm{A}$, which indicates that the source of this disturbance lies on the female side of the cross. The same is true of Family 9.3 except that in this case the unequal ratios are due to an excess of $S_{11}$ over $S_{5}$. In Family 9.4, on the other hand, the disturbance is confined to the male side of the cross, where $S_{1}$ pollen appears to be more successful than that bearing the alternative allele $S_{11}$. Thus, of the six pairwise comparisons that can be made between the four alleles that are segregating in these families, only three have turned out to be significant, two involving the female and one the male side of the crosses giving rise to these families. It is convenient to defer further discussion of what these results may imply until after we have considered the results obtained from the third experiment.

\section{The third experiment}

The results obtained from seven families of Shirley poppies are shown in Table 10. It is at once apparent that the numbers of plants falling into these classes deviate significantly from the expected $1: 1$ ratio in each reciprocal. In the case of reciprocal $\mathrm{A}$, this is due to an excess of plants containing the $S_{2}$ allele, the ratio $S_{1}: S_{2}$ being 219:282 (Table 10), the families being homogeneous in this respect. In reciprocal $\mathrm{B}$, the departure from the expected ratio is due to an excess of plants containing $S_{1}$, the ratio $S_{1}: S_{3}$ being $304: 134$ (Table 10), though in this case, the families are not quite homogeneous in respect of this departure from equality. Since only one of the two kinds of pollen can function in a half-compatible cross, the disturbance in both reciprocals must lie on the female side of the cross. The results from reciprocal A show that female gametophytes containing $S_{2}$ have an advantage over those containing $S_{1}$, and those from reciprocal B show that those containing $S_{1}$ enjoy an advantage over those containing $S_{3}$. This suggests that female gametophytes containing $S_{2}$ should have a selective advantage over those carrying $S_{3}$. The results obtained from three further families of Shirley poppies from the same pedigree as those just considered are consistent with this prediction (Table 11). Thus, from the totals of these families, the ratio of plants containing $S_{2}$ to those containing $S_{3}$ is $111: 58$, which confirms that the rank order of these alleles, in terms of their selective advantage in female gametophytes, is $S_{2}>S_{1}>S_{3}$.

\section{Discussion}

The most surprising feature of the results we have obtained from the three experiments is the ease with 
Table 10 The results obtained from seven families of Shirley poppies; Cross A was $S_{1} S_{2} \times S_{1} S_{3}$ and the reciprocal, Cross B was $S_{1} S_{3} \times S_{1} S_{2}$

\begin{tabular}{cccccc}
\hline & & \multicolumn{3}{c}{ Progeny } \\
\cline { 3 - 5 } Family & Cross & $S_{1} S_{2}$ & $S_{1} S_{3}$ & $S_{2} S_{3}$ & Totals \\
\hline 1 & $\mathrm{~A}$ & - & 47 & 51 & 98 \\
& $\mathrm{~B}$ & 55 & - & 28 & 83 \\
2 & $\mathrm{~A}$ & - & 32 & 32 & 64 \\
& $\mathrm{~B}$ & 48 & - & 12 & 60 \\
3 & $\mathrm{~A}$ & - & 32 & 38 & 70 \\
& $\mathrm{~B}$ & 36 & - & 16 & 52 \\
4 & $\mathrm{~A}$ & - & 41 & 54 & 95 \\
& $\mathrm{~B}$ & 72 & - & 21 & 93 \\
5 & $\mathrm{~A}$ & - & 12 & 27 & 39 \\
& $\mathrm{~B}$ & 29 & - & 11 & 40 \\
6 & $\mathrm{~A}$ & - & 25 & 34 & 59 \\
& $\mathrm{~B}$ & 14 & - & 16 & 30 \\
7 & $\mathrm{~A}$ & - & 30 & 46 & 76 \\
& $\mathrm{~B}$ & 50 & - & 30 & 80 \\
Totals & $\mathrm{A}$ & - & 219 & 282 & 501 \\
& $\mathrm{~B}$ & 304 & - & 134 & 438 \\
\hline
\end{tabular}

The individuals in Families 1 and 2 were raised from seed taken from the same packet, but were grown in different seasons; and similarly for those of Families 3 and 5. Other families originated from different crosses; all families, however, were members of the same pedigree.

For Cross A, the Deviation $\chi_{\{1\}}^{2}=7.922 * *$ and the Heterogeneity $\chi_{[\text {[ }]}^{2}=5.126$.

For Cross B the Deviation $\chi_{[1]}^{2}=65.982^{* * *}$ and the Heterogeneity $\chi_{[6]}^{2}=15.653^{*}$

${ }^{*} P<0.05,{ }^{* *} P<0.01,{ }^{* * *} P<0.001$.

which it has been possible to detect an extra effect of selection acting on the $S$-alleles that are segregating in families of quite modest size. Furthermore, evidence of significantly disturbed segregation ratios is not confined to the wild-type families 9 and 11 , since similar disturbances have been detected in two further R102 families of similar size which, like families 9 and 11 , were segregating for an allele, $S_{15}$, that occurred at a relatively high frequency and three other alleles that occurred at a relatively low frequency in this population (the results from which have not been presented here because their members were not fully classified with respect to their incompatibility phenotype). It could, of course, be argued that we have detected this selection in these wild-type families because they were specially selected for this purpose and that, had we examined a larger number of families chosen at random, few would have exhibited disturbed segregation ratios. However, the evidence from the Shirley families, which were not raised with this purpose in
Table 11 The results obtained from three further families of Shirley poppies which, in relation to those shown in Table 10, arose from Cross C; the parents of Family 10 were plants that had been regenerated from callus culture (Thorlby et al., 1992)

\begin{tabular}{lcrrr}
\hline & & \multicolumn{2}{c}{ Progeny } & \\
\cline { 3 - 4 } Family & Cross & $S_{1} S_{2}$ & $S_{1} S_{3}$ & Totals \\
\hline 8 & $S_{2} S_{3} \times S_{1} S_{3}$ & 55 & 24 & 79 \\
9 & $S_{2} S_{3} \times S_{1} S_{3}$ & 22 & 18 & 40 \\
10 & $S_{2} S_{3} \times S_{1} S_{2}$ & 34 & 16 & 50 \\
Totals & & 111 & 58 & 169 \\
\hline
\end{tabular}

The Deviation $\chi_{[1]}^{2}=16.621^{* * *}$ and the Heterogeneity $\chi_{[2]}^{2}=2.688$. $* * * P<0.001$

Table 12 Estimates of the fitnesses of alleles in families in which the segregation ratios were significantly disturbed:(a) Family 9; (b) Family 11; and (c) the Shirley families (although the Shirley allele $S_{3}$ is known to be the same as the R102 allele $S_{11}$ in Family 9, the counterparts of the other Shirley alleles in the $\mathrm{R} 102$ population are unknown)

(a)

\begin{tabular}{lcccc}
\hline & \multicolumn{4}{c}{ Allele } \\
\cline { 2 - 5 } Family & $S_{1}$ & $S_{5}$ & $S_{10}$ & $S_{11}$ \\
\hline $9 \mathrm{~A}+9.0 \mathrm{~A}$ & 1.40 & 0.60 & 1.00 & 1.00 \\
$9.3 \mathrm{~B}$ & 1.00 & 0.58 & 1.00 & 1.42 \\
$9.4 \mathrm{~A}$ & 1.63 & 1.00 & 1.00 & 0.37 \\
\hline
\end{tabular}

(b)

\begin{tabular}{lccccc}
\hline & \multicolumn{5}{c}{ Allele } \\
\cline { 2 - 6 } Family & $S_{3}$ & $S_{4}$ & $S_{16}$ & $S_{19}$ \\
\hline 11 & 1.59 & 0.41 & 0.78 & 1.22 \\
\hline
\end{tabular}

(c)

\begin{tabular}{lccc}
\hline & \multicolumn{3}{c}{ Allele } \\
\cline { 2 - 4 } Cross & $S_{1}$ & $S_{2}$ & $S_{3}$ \\
\hline A & 0.87 & 1.13 & - \\
B & 1.39 & - & 0.61 \\
C & - & 1.31 & 0.69 \\
\hline
\end{tabular}

mind and which can be regarded, therefore, as having been chosen at random, is clearly not consistent with this view. Thus, it is difficult to avoid the conclusion that the $S$-alleles of this species are subject not only to 
the frequency-dependent selection which maintains the polymorphism, but also to an extra effect of selection.

We would not, of course, have detected this extra effect of selection unless it was quite strong. It is worthwhile, therefore, estimating its strength in the usual way. A convenient way of specifying fitness when estimates are obtained from full-sib families that originate from the cross $S_{\mathrm{i}} S_{\mathrm{j}} \times S_{\mathrm{k}} S_{\mathrm{l}}$ is:

$\begin{array}{lcc}\text { Genotype } & \begin{array}{c}\text { Observed } \\ \text { number }\end{array} & \begin{array}{c}\text { Expected } \\ \text { number }\end{array} \\ S_{\mathrm{i}} S_{\mathrm{k}} & n_{1} & n\left(1+s_{1}+s_{2}\right) / 4 \\ S_{\mathrm{i}} S_{1} & n_{2} & n\left(1+s_{1}-s_{2}\right) / 4 \\ S_{\mathrm{j}} S_{\mathrm{k}} & n_{3} & n\left(1-s_{1}+s_{2}\right) / 4 \\ S_{\mathrm{j}} S_{1} & n_{4} & n\left(1-s_{1}-s_{2}\right) / 4 \\ \text { Total } & n & n\end{array}$

where $s_{1}$ is the coefficient of selection in respect of the maternal alleles $S_{\mathrm{i}}$ and $S_{\mathrm{j}}$, and $s_{2}$ is, similarly, the coefficient of selection in respect of the paternal alleles $S_{\mathrm{k}}$ and $S_{1}$. We note that this model has the convenient property that the sum of the fitnesses over genotypes is unity. Estimates of these coefficients may be obtained as follows:

$s_{1}=\left(n_{1}+n_{2}-n_{3}-n_{4}\right) / n$

and

$s_{2}=\left(n_{1}-n_{2}+n_{3}-n_{4}\right) / n$.

Only the maternal alleles need to be considered with two class families.

Estimates of the fitnesses of alleles for pairs whose ratio departed significantly from $1: 1$ are shown in Table 12. There are two points worth making about these estimates. (1) None of the alleles we have examined is selectively neutral in all of the families we have examined. (2) The advantage or disadvantage of an allele appears, in general, to depend on the alternative with which it segregates. Thus, while $S_{1}$ appears to have an advantage over $S_{5}$ in Family 9.0A and a somewhat greater advantage over its alternative, $S_{11}$, in Family $9.4 \mathrm{~A}$, it appears to be selectively equivalent to $S_{10}$ in Family 9.3B. Again, while $S_{11}$ has an advantage over $S_{5}$ in Family 9.3B, it has none over $S_{10}$ in Family $9.0 \mathrm{~A}$ and, as previously noted, is disadvantageous to $S_{1}$ in Family 9.4A. In the Shirley families, however, there is, as was pointed out in the previous section, a consistent relationship between the three alleles that occur in these families such that their rank order, in terms of their selective advantage, is $S_{2}>S_{1}>S_{3}$. However, it is likely that had more alleles been examined in this Shirley cultivar (at least three more have been identified), it would not have been possible to have arranged them in a linear order.
Now, when the hypothesis for which these experiments were designed to test was first advanced, namely, that the unequal allele frequencies in the natural populations we have investigated were due to an extra effect of selection acting on the locus over and above that due to incompatibility, it was thought that only a few alleles could enjoy an advantage, that any extra effect of selection would be confined to the pollen (and, hence, be of the gametic type), and that any advantage would be unconditional (Campbell \& Lawrence, 1981). The results we have obtained from this investigation are clearly at variance with these expectations. Thus, all of the alleles we have examined appear to have an advantage or disadvantage in at least one of the families we have raised. Again, while this selection appears to be of the gametic type in Family 9 and those of the Shirley cultivar, with the exception of Family 9.4A, this appears to involve the alleles from the female side of the cross, rather than the pollen. In Family 11, on the other hand, the extra effect of selection appears to be of the zygotic, rather than the gametic kind. Hence, the advantage or disadvantage of an allele appears, in general, to depend on the allele with which it is segregating, rather than being unconditional, and more than one type of selection appears to be involved. Thus, assuming, not unreasonably, that the extra effect of selection that we have detected in these families also occurs in the population from which the parents of the wild-type families originated, its effect on allele frequencies must be much more complex than we have hitherto supposed.

The occurrence of an extra effect of selection is not, by itself, a sufficient cause of unequal allele frequencies. It is possible, for example, that while a particular allele has a selective advantage over some of the other alleles in the population, it is at a selective disadvantage with the remainder, such that its net advantage when summation takes place over all other alleles is zero. If this were to be the case, the frequency of the allele in the population would be expected to be the same as in the absence of this selection. Only if the allele has a net advantage (or disadvantage), as a result of this selection, would its frequency in the population be expected to be greater (or less) than in its absence. We need to demonstrate that this is indeed the case, therefore, if we are to invoke an additional effect of selection as a cause of the unequal allele frequencies that we have detected in our natural populations. The present data can hardly be regarded as sufficient for this purpose since, for obvious reasons, we have been able to examine only a very small number of pairwise comparisons between alleles. Nevertheless, it is perhaps encouraging that $S_{1}$, one of the alleles that occurs at a relatively high frequency in the R 102 population, appears to have a net selective advantage over 
the three alleles with which it has been compared in Family 9 (Table 12).

The other chief point worth making about these results concerns the strength of this selection. In previous papers, we have pointed out that the strength of the frequency-dependent selection which maintains the polymorphism becomes attenuated as the number of alleles in the population increases (Lane \& Lawrence, 1993; Lawrence et al., 1994). In populations containing 35-40 alleles, as the populations we have examined appear to do (Lawrence et al., 1993), the strength of this frequency-dependent selection must be quite weak. Yet, the fitness estimates given in Table 12 suggest that the extra effect of selection acting on the relatively small number of alleles we have examined can be quite strong. It follows, therefore, that this extra selection is likely to have a much bigger effect on allele frequencies than that involved with incompatibility in populations that contain more than a small number of alleles.

Finally, we must consider the cause of this extra effect of selection. When discussing the results of the first experiment, in which (because the disturbed segregation ratios were homogeneous over reciprocals) the extra selection appeared to be of the zygotic kind, it was suggested that disturbance was caused by the linkage of the $S$-gene to another that controlled seed dormancy. While this possibility may need to be kept in mind with Family 11, it is difficult to see how it could explain the disturbances detected in the second experiment, in which the extra effect of selection appeared to be of the gametic type, and can be ruled out in the case of the Shirley families, whose seed lacks dormancy. Moreover, we have also detected two kinds of gametic selection. Thus, while in Family 9.4A disturbance is confined to the male side of the cross, where $S_{1}$ pollen appears to be competitively superior to that bearing $S_{11}$ (the kind of disturbance anticipated when the extra selection hypothesis was first advanced), in the remaining five families disturbance is confined to the female side of the cross.

These results suggest, therefore, that there are likely to be at least several different causes of the disturbed ratios that we have detected in these experiments. What might these be? Because all individuals are heterozygous for the $S$-locus, any linkage disequilibrium between this and another locus is expected to decay more quickly than in the more usual circumstances, when both loci can be homozygous. If, therefore, the disturbed ratios that we have observed in these experiments are, in fact, caused by linkage of the $S$-gene to others that are the chief target of the extra effect of selection, this linkage must be quite tight to have been detected in families of relatively modest size. In addition to the possibility that the $S$-gene is linked to one or more genes that determine seed dormancy (a possibility to which we shall return in a future paper), it is also possible that it is linked to one or more of the lethal genes that give rise to the albino phenotypes that were observed not only in the inbred families 9.3 and 9.4, but also in the non-inbred families 9.0 and 11 . Though albino seedlings were not found in the other non-inbred wild-type families, 14 and 20 , this evidence, nevertheless, suggests that the frequency of these lethal genes in the R102 population is surprisingly high.

There are, however, two problems with this evidence. (1) While it has been suggested that the obligate heterozygosity of the $S$-locus provides a shelter for closely linked lethal genes (Rasmuson, 1980), Leach et al. (1986) have argued that this is possible only when such a gene is completely linked to the $S$-gene. But complete linkage would be indistinguishable from pleiotropy (at least in terms of classical genetical analysis) which, for reasons given by Lawrence et al. (1993), is unlikely on other evidence. (2) With the possible exception of Family 11, the extra effect of selection detected in these families appears to be of the gametic type. Yet, any extra effect of selection that arises from the linkage of the $S$-gene to an albino gene would be detected as selection of the zygotic type, since albinism is a property of zygotes. Thus, unless the albino genes are expressed in the gametophytic, as well as the sporophytic phase of the life cycle of poppies, the occurrence of albino seedlings in Families 9 and 11 does not, unfortunately, provide a sufficient explanation for the disturbed segregation ratios we have detected in these families.

While it is not easy to see what further experiments could be usefully undertaken with the wild-type families, in order to find out the cause of the disturbed segregation ratios we have detected in them, the Shirley families are rather more promising in this respect. Two possibilities come to mind. (1) If the disturbance is caused by ovule abortion, it might be possible to detect this by examining dissected capsules both before and after pollination. Since, furthermore, the fitnesses of the three alleles in this material fall into a rank order, it should also be possible to predict the magnitude of ovule abortion and, hence, to correlate ovule abortion with allelic fitness.

(2) It could be investigated whether, as these results suggest, the frequencies of these alleles would be unequal in a population established from approximately equal numbers of plants of the three genotypes, the population being allowed, thereafter, to set seed by open pollination. While the calculations of Leach et al. (1986) indicate that linkage equilibrium between the alleles at the $S$-locus and those of a second gene is 
achieved fairly quickly, the magnitudes of the fitness differences detected in the Shirley families suggest that it should be fairly easy to detect differences in $S$-allele frequencies in the early generations before equilibrium is established.

\section{Acknowledgements}

The work described in this paper was supported by the award of an NERC Research Studentship to V.E.F.-T., which is gratefully acknowledged.

\section{References}

CAMPBell, J. M. AND LAWRENCE, M. J. 1981. The population genetics of the self-incompatibility polymorphism in Papaver rhoeas. II. The number and frequency of S-alleles in a natural population (R106). Heredity, 46, 81-90.

FOOTE, H. C., RIDE, J. P., FRANKLIN-TONG, V. E., WALKER, E, LAWRENCE, M. J. AND FRANKLIN, F. C. H. 1994. Cloning and expression of a novel class of self-incompatibility (S-) gene from Papaver rhoeas. Proc. Natl. Acad. Sci. U.S.A. (in press).

FRANKLIN-TONG, v. E. AND FRANKLIN, F. C. H. 1992. Gametic selfincompatibility in Papaver rhoeas L. Sex. Plant Repro., 5, $1-7$.

FRANKLIN-TONG, V. E., LAWRENCE, M. J. AND FRANKLIN, F. C. H. 1988. An in vitro method for the expression of self-incompatibility in Papaver rhoeas L. using stigmatic extracts. New Phytol., 110, 109-118.

FRANKLIN-TONG, V. E., LAWRENCE, M. J., RUUTH, E. AND FRANKLIN, F. C. H. 1989. Characterisation of a stigmatic component from Papaver rhoeas L. which exhibits the specific activity of a self-incompatibility (S-) gene product. New Phytol., 112, 307-315.

FRANKLIN-TONG, V. E., LAWRENCE, M. J. AND FRANKLIN, F. C. H. 1990. Self-incompatibility in Papaver rhoeas L.: inhibition of incompatible pollen is dependent on pollen gene expression. New Phytol., 116, 319-324.

FRANKLIN-TONG, V. E., ATWAL, K. K., HOWELL, E. C., LAWRENCE, M. J.
AND FRAnKLIN, F. C. H. 1991. Self-incompatibility in Papaver rhoeas $\mathrm{L}$.: there is no evidence for the involvement of stigmatic ribonuclease activity. Plant Cell Env., 14, 423-439.

FRANKLIN-TONG, V. E., RIDE, J. P., READ, N. D., TREWAVAS, A. J. AND FRANKLIN, F. C. H. 1993. The self-incompatibility reaction in Papaver rhoeas is mediated by cytosolic free calcium. Plant J., 4, 163-177.

LANE, M. D. AND LAWRENCE, M. J. 1993. The population genetics of the self-incompatibility polymorphism in Papaver rhoeas. VII. The number of alleles in the species. Heredity, 71, 596-602.

LAWRENCE, M. J. AND O'DONNELL, S. 1981. The population genetics of the self-incompatibility polymorphism in Papaver rhoeas. III. The number and frequency of S-alleles in two further natural populations (R102 and R104). Heredity, 47, 53-61.

LAWRENCE, M. J., AFZAL, M. AND KENRICK, J. 1978. The genetical control of self-incompatibility in Papaver rhoeas. Heredity, 40, 239-253.

LAWRENCE, M. J., LANE, M. D., O'DONNELL, S. AND FRANKLIN-TONG, V. E. 1993. The population genetics of the self-incompatibility polymorphism in Papaver rhoeas. V. The crossclassification of the S-alleles of samples from three natural populations. Heredity, 71, 581-590.

LAWRENCE, M. J., O'DONNELL, S., LANE, M. D. AND MARSHALL, D. F, 1994. The population genetics of the self-incompatibility polymorphism in Papaver rhoeas. VIII. Sampling effects as a possible cause of unequal allele frequencies. Heredity, 72, 345-352.

LEACH, C. R., MAYO, O. AND MORRIS, M. M. 1986. Linkage disequilibrium and gametophytic self-incompatibility. Theor. Appl. Genet., 73, 102-112.

RASMUSON, M. 1980. Linkage to a self-sterility locus. Consequences for segregation and selection. Hereditas, 92 315-319.

THORLBY, G. J., LAWRENCE, M. J. AND FRANKLIN, F. C. H. 1992. Callus culture and plant regeneration in Papaver rhoeas. Arch. Biotech., 1, 1-9.

TONG, v. E. 1986. The Genetics of Self-Incomptability in Papaver rhoeas. Ph.D. thesis, University of Birmingham. 